\title{
Prediction of Substance Concentration in Simulated Moving Bed by Ultraviolet Sensor and Neural Network
}

\author{
I-Chun Chen, ${ }^{1}$ Rey-Chue Hwang, ${ }^{1}$ Chi-Yen Shen, ${ }^{1}$ \\ Shen-Whan Chen, ${ }^{2}$ and Huang-Chu Huang ${ }^{3 *}$ \\ ${ }^{1}$ Department of Electrical Engineering, I-Shou University, \\ No. 1, Sec. 1, Syuecheng Rd., Dashu District, Kaohsiung City 84001, Taiwan \\ ${ }^{2}$ Department of Communication Engineering, I-Shou University, \\ No. 1, Sec. 1, Syuecheng Rd., Dashu District, Kaohsiung City 84001, Taiwan \\ ${ }^{3}$ Department of Electronic Communication Engineering, National Kaohsiung University of Science and Technology, \\ No. 142, Haijhuan Rd., Nanzih District, Kaohsiung City 81157, Taiwan
}

(Received February 4, 2020; accepted April 22, 2020)

Keywords: chromatographic separation, simulated moving bed, UV sensor, neural network

In chromatographic separation, the simulated moving bed (SMB) has been recognized as an important and the cleanest technology. Basically, the SMB is constructed of several columns in series, and can be operated continuously with a fixed switching time. Owing to the adsorption of different substances by the adsorbent, the concentration of the separated substance flowing in the columns changes with time. The success of chromatographic separation will be determined by the time change, substance flow rate, and other possible influencing factors. Therefore, if the concentration of the flowing substance in SMB columns can be sensed immediately, then the accurate control of the SMB can be executed. In this paper, the prediction of substance concentration in SMB chromatographic separation by using an ultraviolet (UV) sensor and a neural network (NN) is presented. In this study, a UV sensor was used to monitor the concentration of the substance, and the NN model was used to predict the substance concentration in real time. The predicted concentration can be used for the real-time control of the moving bed. From the research results shown, it is found that the real-time prediction of substance concentration by the NN based on the sensed UV light intensity can indeed reach a very high accuracy. This result is very promising for the future development of an SMB automatic control mechanism.

\section{Introduction}

Chromatographic separation is an indispensable and important technology in the manufacture of chemical and biomedical products. The separation process uses the difference in adsorption of different substances in the mixture between the stationary phase and the fluid phase to achieve separation. The accuracy of the separation is an indicator of the product's quality and usability. Therefore, how to use an effective and accurate separation technology has always been an important issue to be considered by the chemical and biochemical industries. ${ }^{(1-3)}$

*Corresponding author: e-mail: h4530@nkust.edu.tw https://doi.org/10.18494/SAM.2020.2823 
Currently, the simulated moving bed (SMB) system is recognized as the most advanced and efficient chromatographic separation technology because of its continuous feeding capability. The multiple-column SMB system can be continuously switched at a fixed time, so that its inlet port and outlet port can also be changed constantly. Such a characteristic of the SMB not only improves the efficiency of the adsorbent used in the adsorption bed, but also reduces the consumption of solvent and water in the whole separation process. Thus, SMB separation is also recognized as the cleanest manufacturing technology in chromatography. Figure 1 shows an SMB system with eight columns. ${ }^{(4)}$

To increase the production capacity, the precise and effective control of the multiple-column SMB has always been a challenging problem. ${ }^{(5-10)}$ In fact, the SMB is a very complex and nonlinear system; the current SMB mathematical model is only an approximate model and cannot fully represent the real dynamic behavior and state of the SMB's actual operation. ${ }^{(11-13)}$ Therefore, the aim of this study is to find the actual separation process of an SMB and then construct an effective SMB control mechanism.

In this research, to observe the actual operation condition of the SMB, an ultraviolet (UV) sensor was used to sense the concentration change of the separated substance in the SMB columns. On the basis of the learning capability of the neural network $(\mathrm{NN})$, the relationship between the intensity of UV light and the substance concentration is obtained by the NN. That means that a well-trained NN model can accurately predict the concentration of an individual substance in the SMB. In other words, such a well-trained NN model could replace a real SMB system, enabling the development of an effective and precise SMB control mechanism, and then the accuracy of the SMB in the chromatographic separation could be improved. Figure 2 shows the whole SMB control process desired to be developed.

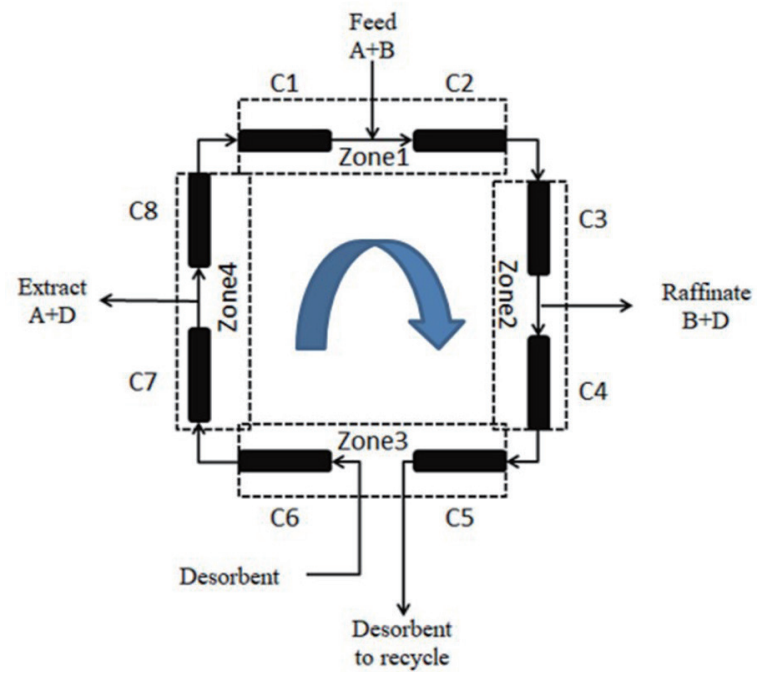

Fig. 1. (Color online) SMB system with eight columns.

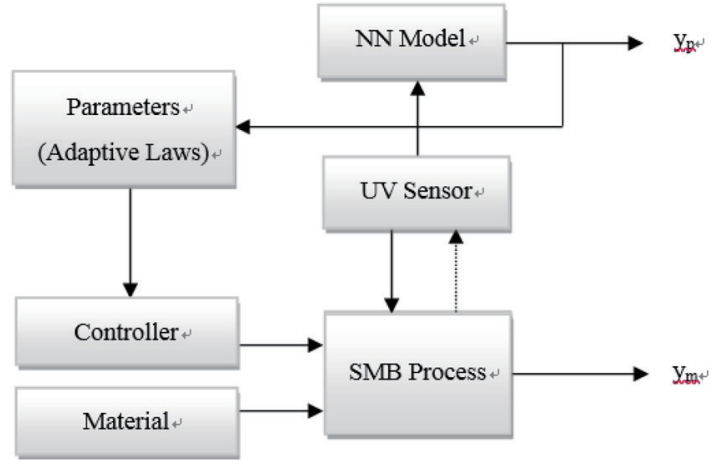

Fig. 2. Whole SMB control process. 


\section{NN Model}

This research is expected to lead to the development of an accurate and practical NN model for the SMB separation process. Owing to the excellent self-learning and self-tuning abilities, the NN technique has been widely used in various applications, ${ }^{(14-18)}$ especially signal processing and control engineering. Through the training of historical data, the weights of the NN are adjusted to fit the data appropriately. The mapping model of input/output data can be automatically developed while the learning process of the NN is finished. Then, the welltrained $\mathrm{NN}$ can be used as a smart signal processor for classification, identification, prediction, control, and so forth.

The NN structure commonly known as a multilayered feedforward network was used in this study. The three-layer feedforward NN architecture shown in Fig. 3 is the selected topology, and the backpropagation (BP) learning algorithm is used to train the NN. ${ }^{(17)}$

\section{Experiments}

\subsection{Substance concentration vs UV light intensity}

To observe the response of solutions with different concentrations to UV light, experiments on mixed and pure solutions were first performed. Table 1 lists the ten mixtures of two substances, A (salicylic acid) and B (caffeine), with different concentrations used in the experiment. Table 2 lists the concentrations of the two pure solutions, A and B, used in the experiment. Figure 4 displays the variations of the light intensity of each mixture under different wavelengths of UV irradiation. Figures 5 and 6 present the light intensity variations of solutions $\mathrm{A}$ and $\mathrm{B}$ under different wavelengths of $\mathrm{UV}$ irradiation, respectively.

From the experimental results shown, it is clearly found that the substances of different concentrations have different light intensity responses under the different wavelengths of UV light. This means that the substance concentration can be predicted from the light intensity.

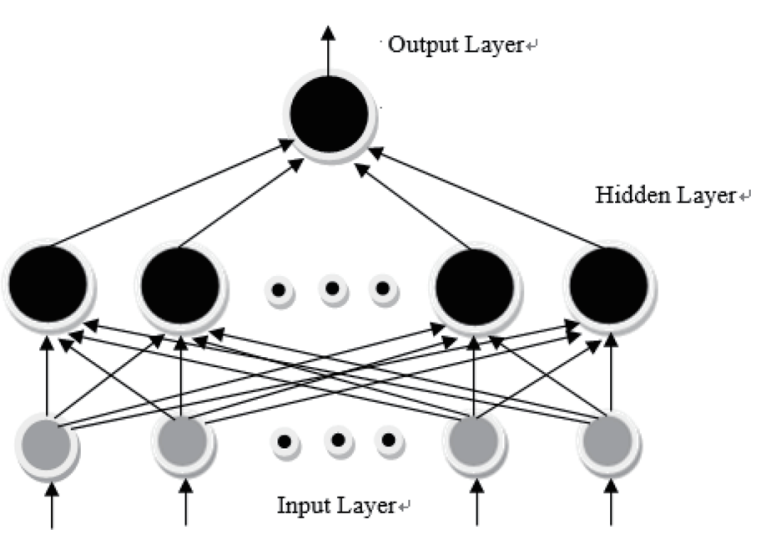

Fig. 3. A three-layer feedforward NN.
Table 1

Mixtures of two substances, A and B.

\begin{tabular}{rcc}
\hline & \multicolumn{2}{c}{ Mixture } \\
\cline { 2 - 3 } No. & $\begin{array}{c}\text { Concentration of A } \\
(\mathrm{ppm})\end{array}$ & $\begin{array}{c}\text { Concentration of B } \\
(\mathrm{ppm})\end{array}$ \\
\hline 6 & 1750 & 250 \\
15 & 3250 & 500 \\
18 & 3750 & 500 \\
22 & 3325 & 500 \\
31 & 1750 & 750 \\
34 & 1250 & 750 \\
39 & 2000 & 500 \\
42 & 1500 & 750 \\
44 & 500 & 750 \\
52 & 750 & 500 \\
\hline
\end{tabular}


Table 2

Concentrations of two pure solutions, A and B.

\begin{tabular}{lccc}
\hline No. & Concentration of solution A (ppm) & No. & Concentration of solution B (ppm) \\
\hline A-2 & 2535 & B-2 & 2500 \\
A-5 & 1014 & B-5 & 1000 \\
A-10 & 507 & B-10 & 500 \\
A-20 & 253.5 & B-20 & 1666.7 \\
\hline
\end{tabular}

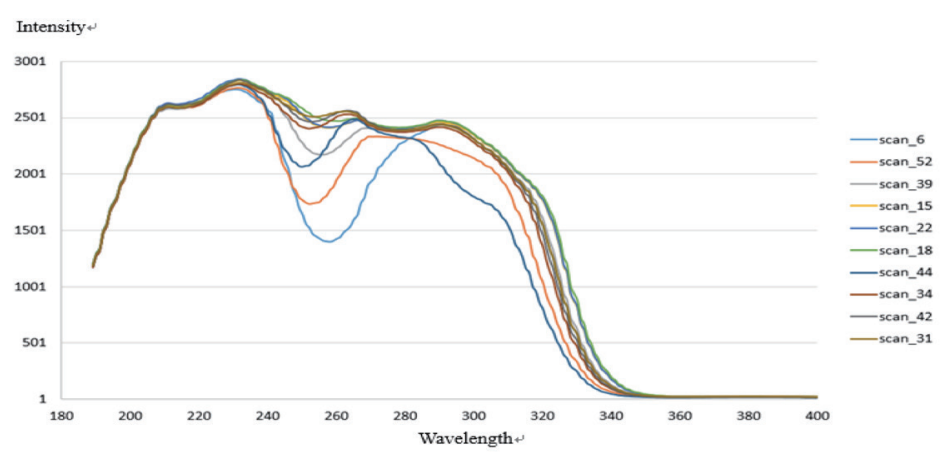

Fig. 4. (Color online) Variations of light intensity of mixtures under different wavelengths of UV irradiation.

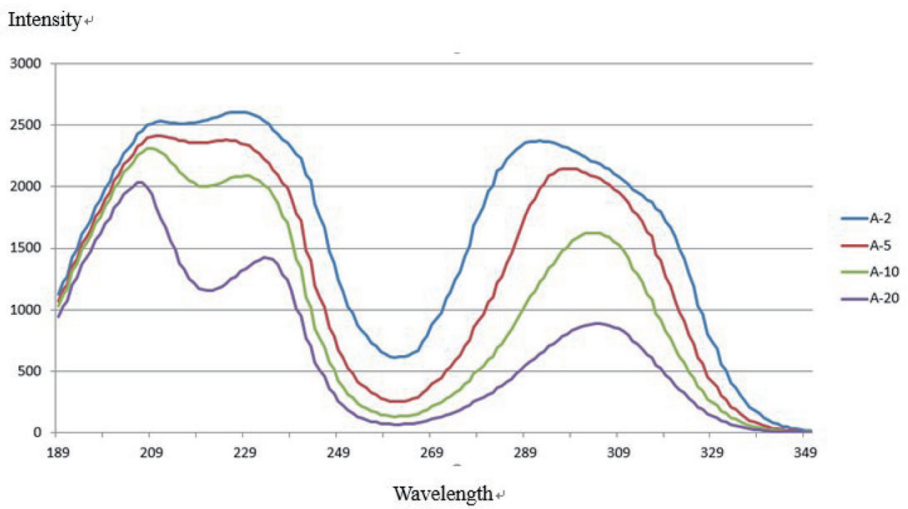

Fig. 5. (Color online) Light intensity variations of solutions A under different wavelengths of UV irradiation.

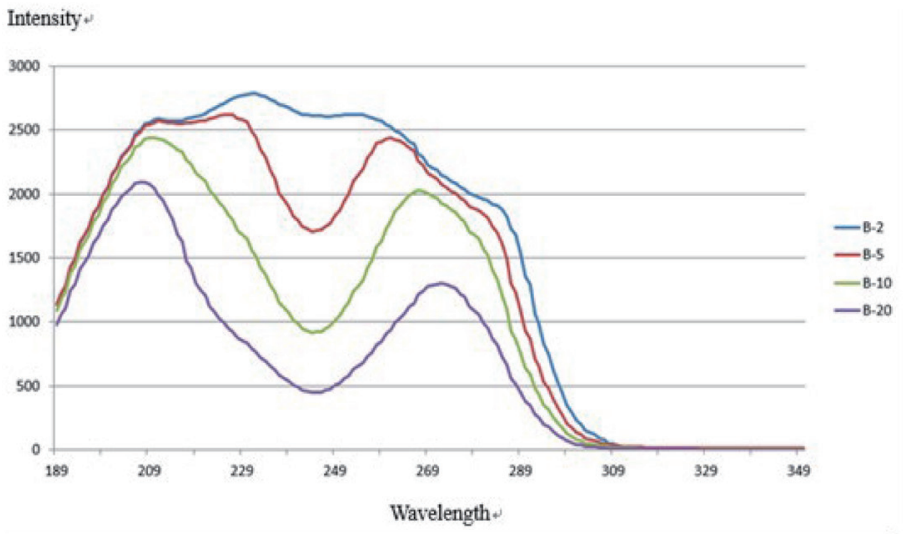

Fig. 6. (Color online) Light intensity variations of solutions B under different wavelengths of UV irradiation. 


\subsection{NN prediction}

In this research, the prediction of individual substance concentrations in mixtures with two components (salicylic acid and caffeine) by using the NN was studied. To collect the light intensity of different mixtures under different wavelengths of UV radiation, 64 mixtures with different salicylic acid and caffeine concentrations were investigated experimentally and a total of 64 data sets were obtained. Table 3 lists some examples of the experimental results. To ensure the correctness of the NN model in this study, the 64 data sets were organized randomly to generate five different data groups, i.e., Group-1 to Group-5. For each group, 42 data sets were used for NN training and 22 data sets were used for testing. The size of the NN was 12060-60-1. The 120 inputs were all UV light intensity values. The mean absolute percentage error (MAPE) was used evaluate prediction results. Table 4 shows the MAPEs of the predicted concentrations of salicylic acid and caffeine obtained by the well-trained NN model.

Table 3

Light intensities of different mixtures under different wavelengths of UV radiation.

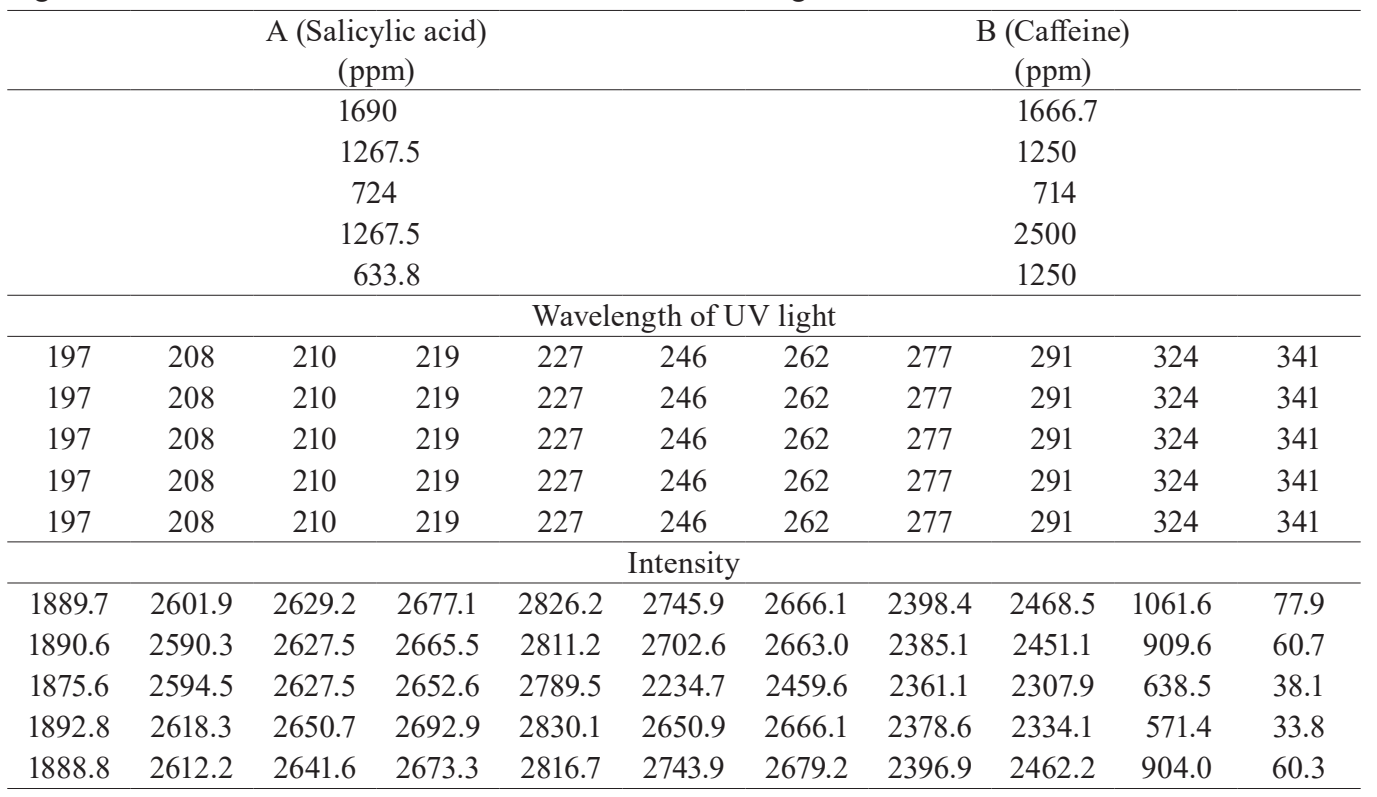

Table 4

MAPEs of predicted concentrations of salicylic acid and caffeine.

\begin{tabular}{lcccc}
\hline & \multicolumn{2}{c}{ Salicylic acid } & \multicolumn{2}{c}{ Caffeine } \\
\cline { 2 - 5 } & $\begin{array}{c}\text { Training } \\
\text { (MAPE) (\%) }\end{array}$ & $\begin{array}{c}\text { Test } \\
\text { (MAPE) (\%) }\end{array}$ & $\begin{array}{c}\text { Training } \\
\text { (MAPE) (\%) }\end{array}$ & $\begin{array}{c}\text { Test } \\
\text { (MAPE) (\%) }\end{array}$ \\
\hline Group-1 & 1.11 & 1.21 & 1.27 & 1.31 \\
Group-2 & 1.26 & 1.28 & 1.24 & 1.35 \\
Group-3 & 1.31 & 1.28 & 1.16 & 1.36 \\
Group-4 & 1.32 & 1.36 & 1.33 & 1.24 \\
Group-5 & 1.18 & 1.30 & 1.29 & 1.35 \\
\hline Avg. & 1.24 & 1.29 & 1.26 & 1.32 \\
\hline
\end{tabular}


Figures 7(a)-7(e) are superposition diagrams of salicylic acid concentrations predicted by the NN for the data in Group-1 to Group-5, and superposition diagrams of caffeine concentrations predicted by the NN for the data in Group-1 to Group-5 are shown in Figs. 8(a)-8(e).

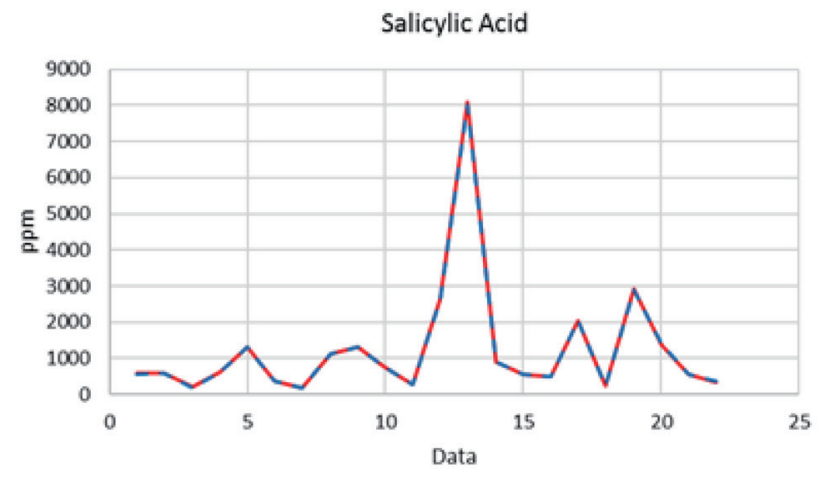

(a)

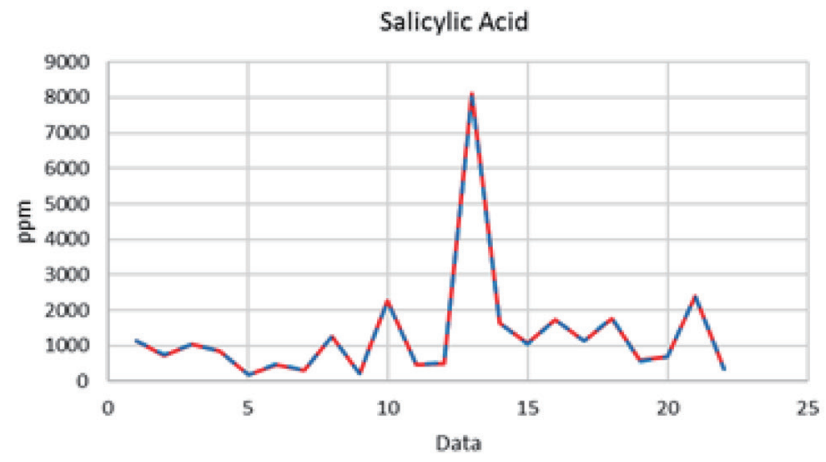

(c)

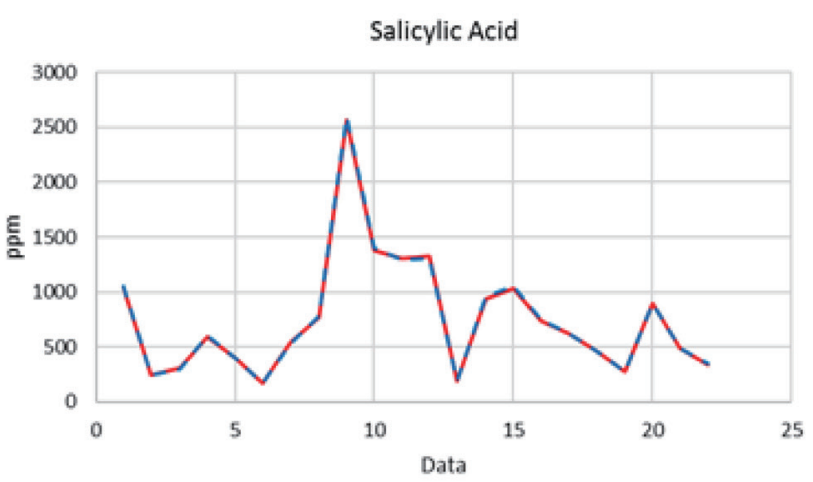

(b)

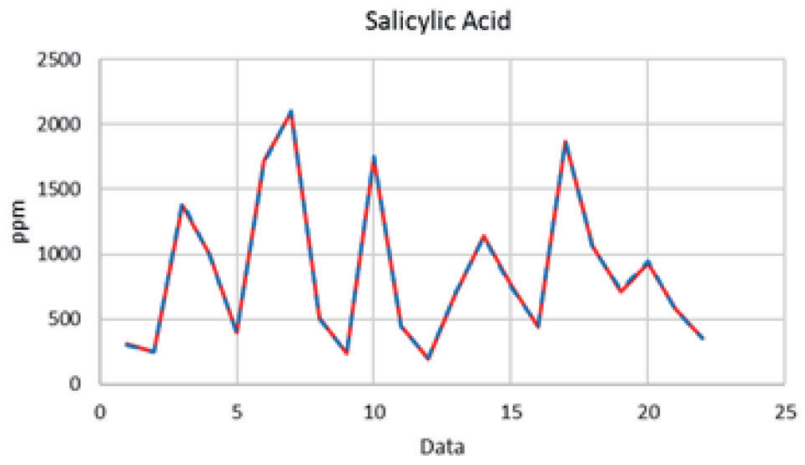

(d)

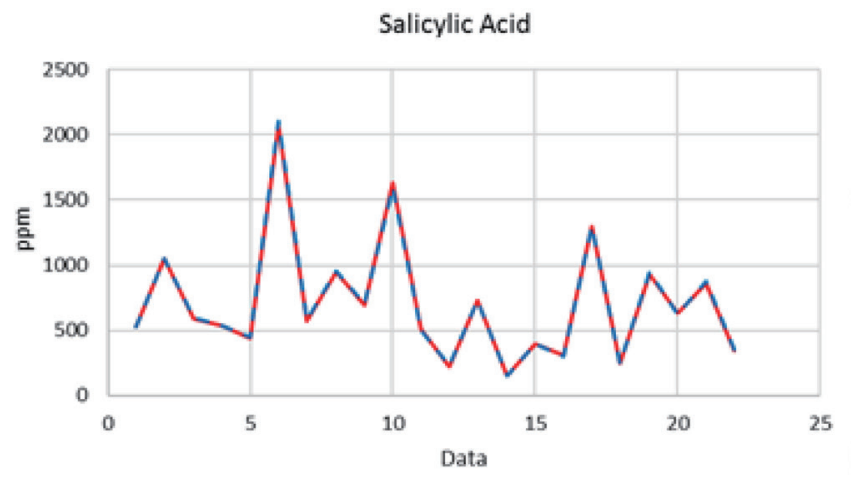

(e)

Fig. 7. (Color online) Concentrations of salicylic acid predicted by NN for data in Group-1 to Group-5. (Solid line: Desired value. Dashed line: Predicted value) (a) Group 1. (b) Group 2. (c) Group 3. (d) Group 4. (e) Group 5. 
Caffeine

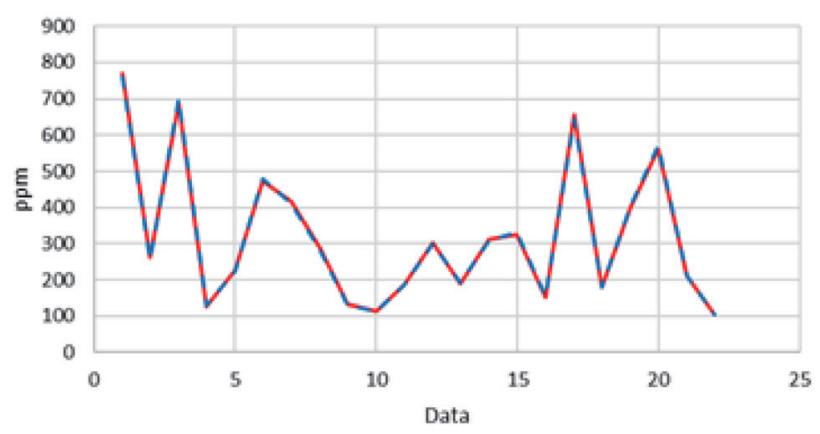

(a)

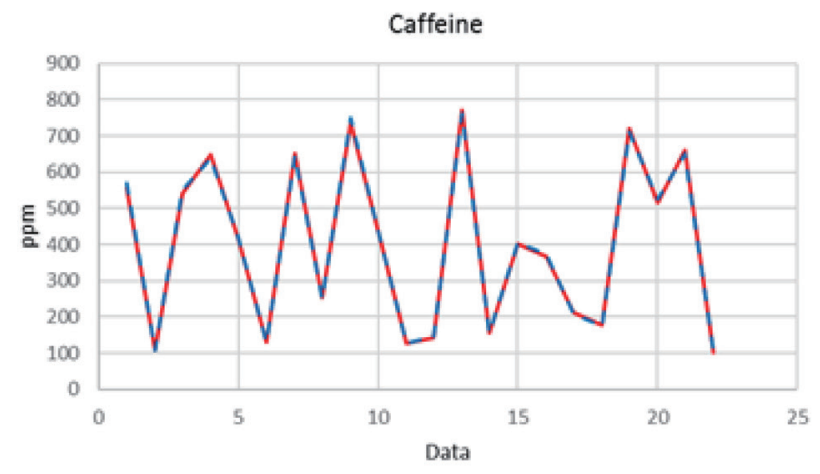

(c)

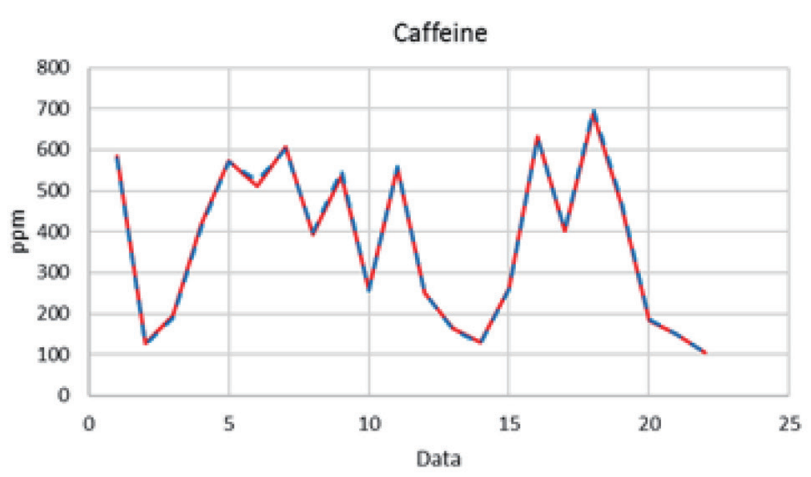

(b)

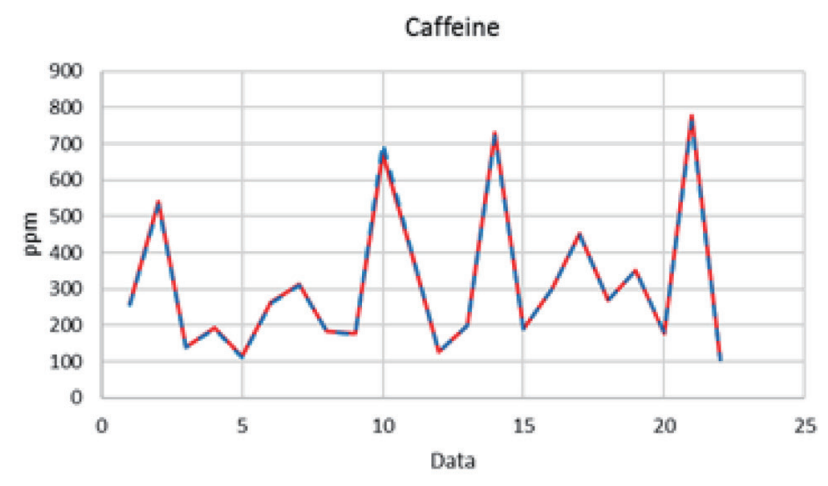

(d)

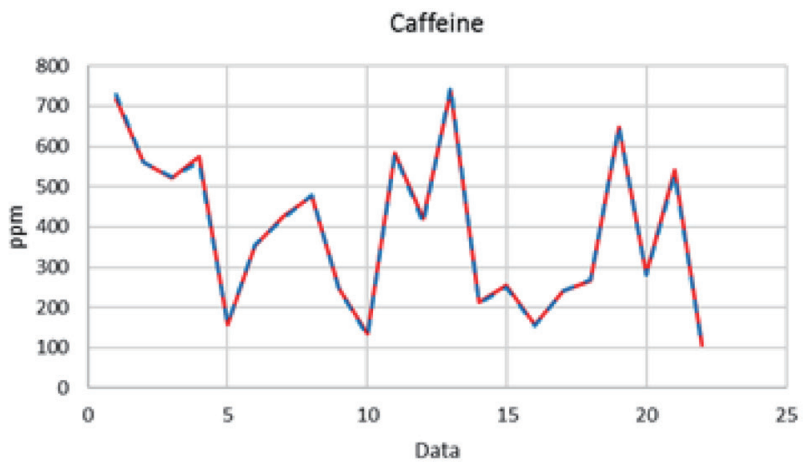

(e)

Fig. 8. (Color online) Concentrations of caffeine predicted by NN for data in Group-1 to Group-5. (Solid line: Desired value. Dashed line: Predicted value) (a) Group 1. (b) Group 2. (c) Group 3. (d) Group 4. (e) Group 5.

\section{Conclusions}

In this research, a substance concentration prediction mechanism for the SMB operation process was developed. This prediction mechanism was constructed using a UV sensor and an NN model. The UV sensor is used to sense the light intensity of the substance and the NN 
model is used to predict the substance concentration in real time. To demonstrate the feasibility of NN technology for the prediction of substance concentration, the intensity of UV light vs the substance concentration for different wavelengths was investigated experimentally. These intensity data were then used to train and test the NN. From the research results shown, it was clearly found that the real-time prediction of substance concentration by the NN based on the sensed UV light intensity indeed can reach very high accuracy. The total average MAPE values for salicylic acid and caffeine predictions in five data groups were 1.29 and $1.32 \%$, respectively. This result is very promising for the future development of an SMB automatic control mechanism.

\section{Acknowledgments}

This research was supported by the Ministry of Science and Technology, Taiwan, under contract No. MOST-107-2221-E-214-029.

\section{References}

1 C. Migliorini, M. Mazzotti, and M. Morbidelli: J. Chromatogr. A 827 (1998) 161. https://doi.org/10.1016/S00219673(98)00643-8

2 M. Mazzotti, G. Storti, and M. Morbidelli: J. Chromatogr. A 786 (1997) 309. https://doi.org/10.1016/S00219673(97)00594-3

3 O. D. Giovanni, M. Mazzotti, M. Morbidelli, F. Denet, W. Hauck, and R. M. Nicoud: J. Chromatogr. A 919 (2001) 1. https://doi.org/10.1016/S0021-9673(01)00799-3

4 S. Peper, M. Johannsen, and G. Brunner: J. Chromatogr. A 1176 (2007) 246. https://doi.org/10.1016/ j.chroma.2007.09.090

5 M. Alamir, F. Ibrahim, and J. P. Corriou: J. Process Control 16 (2006) 333. https://doi.org/10.1016/ j.jprocont.2005.07.001

6 G. Erdem, S. Abel, M. Morari, M. Mazzotti, M. Morbidelli, and J. H. Lee: Ind. Eng. Chem. Res. 43 (2004) 405. https://doi.org/10.1021/ie030377o

7 S. Natarajan and J. H. Lee: Comput. Chem. Eng. 24 (2000) 1127. https://doi.org/10.1016/S0098-1354(00)004932

8 I. H. Song, S. B. Lee, H. K Rhee, and M. Mazzotti: Chem. Eng. Sci. 61 (2006) 1973. https://doi.org/10.1016/ j.ces.2005.10.010

9 P. Suvarov, A. Vande Wouwer, and A. Kienle: Proc. 8th IFAC Int. Symp. Advanced Control of Chemical Processes 45 (2012) 137. https://doi.org/10.3182/20120710-4-SG-2026.00098

10 B. Medi, K. M. Khoda and M. Amanullah: Ind. Eng. Chem. Res. 54 (2015) 6527. https://doi.org/10.1021/acs. iecr.5b00553

11 L. Li, D. C. Yuan, and Y. W. Jing: Proc. 2010 Int. Conf. Intelligent Control and Information Processing No. PART 1, (2010) 497. http://doi.org/10.1109/CCDC.2010.5498455

12 G. Sharma, S. V. Vignesh, K. Hariprasad, and S. Bhartiya: IFAC-Papers 48 (2015) 477. https://doi.org/10.1016/ j.ifacol.2015.09.013

13 J. Lu: Proc. World Congr. Intelligent Control and Automation (WCICA) (2004) 3488. http://doi.org/10.1109/ WCICA.2004.1343194

14 D. Yang, T. Jiaxin, Z. Binghong, and G. Fei: Proc. 2017 Int. Conf. Computer Systems, Electronics and Control (2018) 1081. http://doi.org/10.1109/ICCSEC.2017.8446886

15 H. Alibi, F. Fayala, A. Jemni, and X. Zeng: Spec. Top. Rev. Porous Media 3 (2012) 35. https://oi.org/10.1615/ SpecialTopicsRevPorousMedia.v3.i1.40

16 W. Z. Cui, C. C. Zhu, and H. P. Zhau: Thin Solid Films 473 (2005) 224. https://doi.org/10.1016/j.tsf.2004.06.121

17 C. Y. Shen, H. C. Huang, and R. C. Hwang: Sens. Actuators, A 147 (2008) 464. https://doi.org/10.1016/ j.sna.2008.05.025

18 C. Y. Shen, H. C. Huang, and R. C. Hwang: J. Chemom. 22 (2008) 548. https://doi.org/10.1002/cem.1183 


\section{About the Authors}

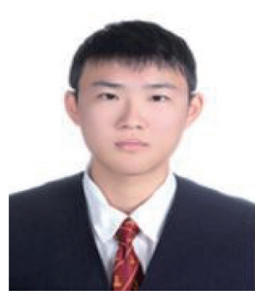

I-Chun Chen graduated from the Department of Electrical Engineering of I-Shou University. Currently, he is pursuing his Ph.D. degree in electrical engineering. His research interests are in artificial intelligence, fuzzy control, and signal processing. (qe660212@gmail.com)

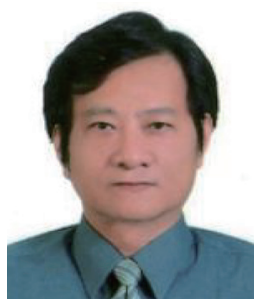

Rey-Chue Hwang received his Ph.D. degree in electrical engineering from Southern Methodist University, Dallas, TX, in 1993. Currently, he is a full professor of the Electrical Engineering Department, I-Shou University, Taiwan, R.O.C. Dr. Hwang has published more than 300 papers in various journals and conferences in the areas of artificial intelligence systems, signal processing, and fuzzy control. He is now a fellow of IET and a senior member of IEEE.

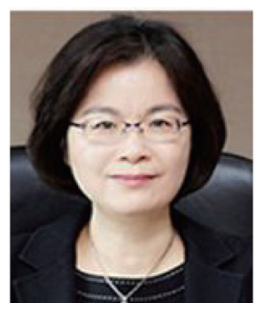

Chi-Yen Shen received her B.S. degree in electrical engineering from Cheng-Kung University in Taiwan in 1987 and her Ph.D. degree in electrical engineering from National Sun Yat-Sen University, Taiwan, in 1991. She is currently a professor of electrical engineering at I-Shou University, Taiwan. Her research interests are in surface acoustic wave devices, sensors, and electronic ceramics. (cyshen@isu.edu.tw)

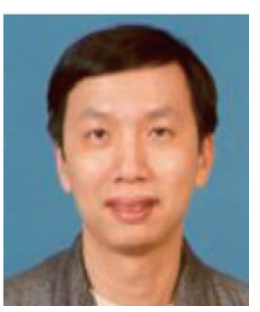

Shen-Whan Chen received his Ph.D. degree in electrical engineering from the University of Illinois at Chicago, USA, in 1994. He has worked for Delco, MA/COM, and Hughes Space and Comm. He is currently with the Communication Engineering Department, I-Shou University, Kao-Shung, Taiwan, as an associate professor. His main research interests are in RF circuit design and AI signal processing. (jasonchen@isu.edu.tw)

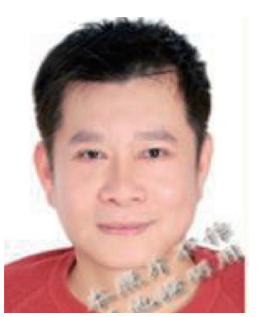

Huang-Chu Huang received his Ph.D. degree in electrical engineering from National Sun Yat-Sen University, Taiwan, in 2001. Currently, he is a professor of the Electronic Communication Department, National Kaohsiung University of Science and Technology, Kaohsiung City, Taiwan. His research interests are in the areas of control, power signal prediction, and neural network applications. (h4530@nkust.edu.tw) 
